EPJ Web of Conferences 20, 03002 (2012)

DOI: $10.1051 /$ epjconf/20122003002

(C) Owned by the authors, published by EDP Sciences, 2012

\title{
An effective thermodynamic potential from the instanton vacuum with the Polyakov loop
}

\author{
Seung-il Nam ${ }^{\mathrm{a}}$ \\ School of Physics, Korea Institute for Advanced Study, Seoul 130-722, Republic of Korea
}

\begin{abstract}
In this talk, we report our recent studies on an effective thermodynamic potential $\left(\Omega_{\text {eff }}\right)$ at finite temperature $(T \neq 0)$ and zero quark-chemical potential $\left(\mu_{\mathrm{R}}=0\right)$, using the singular-gauge instanton solution and Matsubara formula for $N_{c}=3$ and $N_{f}=2$ in the chiral limit, i.e. $m_{q}=0$. The momentum-dependent constituent-quark mass is computed as a function of $T$, together with the Harrington-Shepard caloron solution in the large- $N_{c}$ limit. In addition, we take into account the imaginary quark-chemical potential $\mu_{\mathrm{I}} \equiv A_{4}$, identified as the traced Polyakov-loop $(\Phi)$ as an order parameter for the $\mathbb{Z}\left(N_{c}\right)$ symmetry, characterizing the confinement (intact) and deconfinement (spontaneously broken) phases. As a consequence, we observe the crossover of the chiral $(\chi)$ order parameter $\sigma^{2}$ and $\Phi$. It also turns out that the critical temperature for the deconfinement phase transition, $T_{c}^{\mathbb{Z}}$ is lowered by about $(5 \sim 10) \%$ in comparison to the case with the constant constituent-quark mass. This behavior can be understood by considerable effects from the partial chiral restoration and nontrivial QCD vacuum on the $\Phi$. Numerical results show that the crossover transitions occur at $\left(T_{c}^{\chi}, T_{c}^{\mathbb{Z}}\right) \approx(216,227) \mathrm{MeV}$.
\end{abstract}

\section{Introduction}

We note that the phase structure of quantum chromodynamics (QCD), as a function of temperature $T$ and quark-chemical potential $\mu$, represents the breaking patterns of the relevant symmetries in QCD. Simultaneously, each QCD phase can be characterized by the corresponding order parameters, reflecting the nature of the symmetries. In this sense, exploring the QCD phase diagram is of great importance in understanding strongly interacting systems. Especially, recent energetic progresses, achieved in the ultra-high energy experimental facilities, such as the RHIC, have triggered much interest to investigate the QCD phase structure in the vicinity of high $T \approx T_{c}$, whereas $\mu$ remains relatively small, being similar to the early universe.

Starting from the first principle, the lattice QCD (LQCD) simulations must be a promising method to investigate this region ( $T \neq 0$ and $\mu \approx 0$ ) with less difficulties, such as the sign problem [1-6]. Many attempts have been also done in various effective field-theoretical approaches [7-11,14,12,13,1519]. Among them, interestingly enough, the Polyakov-loop-augmented Nambu-Jona-Lasinio (pNJL) model describes the crossover of the two different QCD order parameters for the chiral and $\mathbb{Z}\left(N_{c}\right)$ symmetries, represented by the chiral condensate $\langle\bar{q} q\rangle \propto \sigma^{2}$ and the traced Polyakov loop $\langle\phi\rangle \equiv \Phi$, respectively [7-11,14]. If the $\mathbb{Z}\left(N_{c}\right)$ symmetry is intact, $\Phi$ becomes zero, indicating the confinement phase. On the contrary, provided that the symmetry is broken spontaneously, one has $\Phi \neq 0$ for the deconfinement one.

Instanton model can be also thought as an appropriate framework to be employed for this finite- $T$ subject, considering that it has provided remarkable descriptions so far for various nonperturbative QCD and hadron properties. Note that the instanton solution at finite $T$, being periodic in Euclidean time, i.e. caloron turned out to be essential for this purpose [20,21]. Nonetheless for its relevance,

a e-mail: sinam@kias.re.kr

This is an Open Access article distributed under the terms of the Creative Commons Attribution-Noncommercial License 3.0, which permits unrestricted use, distribution, and reproduction in any noncommercial medium, provided the original work is properly cited. 


\section{EPJ Web of Conferences}

its practical application is still under development [26,27,22-25]. Confinement properties have been discussed as well with semi-classical objects, such as the meron (a half of regular-gauge instanton), by indicating the area law for the Wilson loop [28,29]. The caloron with non-trivial holonomy, so called the Kraan-van Baal-Lee-Lu (KvBLL) caloron [20,21], was taken into account as a lump of dyons to understand the QCD confinement [22,23,25].

In the present talk, we want to develop an effective thermodynamic potential $\left(\Omega_{\text {eff }}\right)$ at finite $T$ with $\mu_{\mathrm{R}}=0$, employing the instanton framework. Our strategy is rather simple and practical as follows:

i) Using the instanton distribution function at finite $T$ from the caloron solution with trivial holonomy (the Harrington-Shepard caloron) [30,31], we first compute the instanton density and average size of instanton as functions of $T$, resulting in that the instanton effect remains finite even beyond the critical temperature $T_{c} \sim \Lambda_{\mathrm{QCD}}$. Taking into account these ingredients, we finally obtain ( $\boldsymbol{k}$ (three momentum), $T$ (temperature))-dependent constituent-quark mass $M, M_{k, T}$, which plays the most important role in the present approach.

ii) In constructing $\Omega_{\text {eff }}$, we take into account a practical way, instead of using the caloron and its quark zero-mode solution: $\Omega_{\mathrm{eff}}$ is obtained by applying the Matsubara formula to the effective action, which is derived from the usual singular-gauge instanton solution at $T=0$, as done usually in effective models [15-19,7-11,14].

iii) The singular-gauge instanton solution is nothing to do with the confinement [29]. On the contrary, it explains the nonperturbative QCD properties very well in terms of the spontaneous breakdown of chiral symmetry $(\mathrm{SB} \chi \mathrm{S})$. Hence, considering the chiral and $\mathbb{Z}\left(N_{c}\right)$ symmetries on the same footing as in the pNJL model, we introduce the imaginary quark-chemical potential $\mu_{\mathrm{I}} \equiv A_{4}$, which corresponds to the uniform color gauge field in the Polyakov gauge. It will be identified later as the traced Polyakov loop $\Phi$, as an order parameter for the spontaneous breakdown of $\mathbb{Z}\left(N_{c}\right)$ symmetry, i.e. the deconfinement phase transition.

Considering all these ingredients, now we can write a neat expression for $\Omega_{\text {eff }}$ as a function of $T$ with $M_{k, T}$. By solving the saddle-point equations with respect to the mean fields, i.e. $\sigma$ and $\Phi$, we can draw the curves for $\sigma^{2}$ and $\Phi$ as functions of $T$. From the numerical calculations, we observe that the first-order deconfinement phase transition in pure-glue QCD is modified to the crossover one, according to the mixing of dynamical quarks and $\Phi$ in $\Omega_{\text {eff }}$. In contrast, the mixing gives only negligible modifications on $\sigma^{2}$. As a result, the crossover of the two different QCD order parameters is shown by the mixing. It also turns out that $T_{c}$ for the deconfinement phase transition $\left(T_{c}^{\mathbb{Z}}\right)$ is lowered by about $10 \%$ with $M_{k, T}$, in comparison to the case with a constant $M\left(M_{0,0} \approx 350 \mathrm{MeV}\right)$. From this observation, we can conclude that the nontrivial QCD vacuum contribution and partial chiral restoration play a considerable role even for deconfinement phase transition. The numerical results show that $T_{c}^{\chi}=216 \mathrm{MeV}$ and $T_{c}^{\mathbb{Z}}=227 \mathrm{MeV}$. The discrepancy between them becomes about $10 \mathrm{MeV}$, which is rather larger than that computed in the local pNJL model [8-10]. We note that the LQCD simulations provides smaller values than ours, whereas $T_{c}^{\mathbb{Z}}$ is comparable [1-6]. We also find that $\sigma^{2}$ depends much on the partial chiral restoration.

We organize the present report as follows: In Section 2, we briefly explain the theoretical framework for obtaining the effective thermodynamic potential. The numerical results with discussions are given in Section 3. The final Section is devoted to the summary and outlook. We note that this talk is based on Ref. [32], and more details on the theoretical evaluations can be found there.

\section{Theoretical framework}

In the present section, we drive an effective thermodynamic potential $\Omega_{\text {eff }}$, considering all the ingredients discussed in Section II and III of Ref. [32]. In addition, we take into account the imaginary quark chemical potential $\left(\mu_{\mathrm{I}} \equiv A_{4}\right)$, which will be identified as the traced Polyakov loop as an order parameter for the deconfinement phase transition [7,14]. Moreover, this corresponds to the uniform color gauge field, induced in the Polyakov gauge, $A_{\mu}=\left(\mathbf{0}, A_{4}\right)$. All calculations will be performed in the case for $N_{c}=3, N_{f}=2$, and $\mu_{\mathrm{R}}=0$ in the chiral limit for the leading large- $N_{c}$ contributions. In order to evaluate $\Omega_{\text {eff }}$ as a function of $T$ from the effective action, we employ the anti-periodic Matsubara 
formula for fermions in Euclidean space:

$$
\int \frac{d^{4} k}{(2 \pi)^{4}} f\left(\boldsymbol{k}, k_{4}\right) \rightarrow T \sum_{n=-\infty}^{\infty} \int \frac{d^{3} \boldsymbol{k}}{(2 \pi)^{3}} f\left(\boldsymbol{k}, w_{n}\right),
$$

where the Matsubara frequency reads $w_{n}=(2 n+1) \pi T$. We also include an imaginary quark-chemical potential $[7,10]$, which can be identified as the fourth component of $\mathrm{SU}\left(N_{c}\right)$ gauge field $\left(A_{4}\right)$ in Euclidean space, resulting in a simple replacement $k \rightarrow k-A_{4}$ in $\Omega_{\text {eff }}$. Using these ingredients, we can have the following effective thermodynamic potential per unit volume in the presence of the dynamical quarks and $A_{4}$ :

$$
\Omega_{\mathrm{eff}}^{\mathrm{q}+\mathrm{A}_{4}}=2 \sigma^{2}-N_{f} T \sum_{n=-\infty}^{\infty} \int \frac{d^{3} \boldsymbol{k}}{(2 \pi)^{3}} \operatorname{Tr}_{c} \ln \left[\frac{\left(k-A_{4}\right)^{2}+M_{\boldsymbol{k}, T}^{2}}{T^{2}}\right],
$$

where the $\sigma$ is a function of $T$ as well. Note that we have ignored $A_{4}$ in $M$ for simplicity. After a tedious but straightforward manipulation, one is led to the following expression:

$$
\Omega_{\mathrm{eff}}^{\mathrm{q}+\mathrm{A}_{4}} \approx 2 \sigma^{2}-N_{f} T \int \frac{d^{3} \boldsymbol{k}}{(2 \pi)^{3}} \operatorname{Tr}_{c}\left[\frac{E_{\boldsymbol{k}, T}}{T}+\ln \left[\left(1+e^{-\frac{E_{\boldsymbol{k}, T}-\mathrm{A}_{4}}{T}}\right)\left(1+e^{-\frac{E_{\boldsymbol{k}, T}+\mathrm{A}_{4}}{T}}\right)\right]\right] .
$$

Here, we have used that

$$
E_{k, T}=\left(\boldsymbol{k}^{2}+M_{\boldsymbol{k}, T}^{2}\right)^{1 / 2}, \quad M_{\boldsymbol{k}, T}=M_{0, T}\left[\frac{2}{2+\boldsymbol{k}^{2} \bar{\rho}^{2}}\right]^{2},
$$

where the $M_{0, T}$ is the $T$-dependent constituent-quark mass for $\boldsymbol{k}=0$. For more details, see also Appendix in Ref. [32].

Now, we are in a position to consider the traced Polyakov loop $\phi$, defined in a $\mathrm{SU}\left(N_{c}\right)$ gauge group as:

$$
\phi=\frac{1}{N_{c}} \operatorname{Tr}_{c} \exp \left(\frac{i A_{4}}{T}\right), \quad \phi^{*}=\frac{1}{N_{c}} \operatorname{Tr}_{c} \exp \left(\frac{-i A_{4}}{T}\right) .
$$

Taking into account the Polyakov gauge, $A_{4}$ is diagonal in a $N_{c} \times N_{c}$ matrix. For instance, the perturbative YM potential can be expressed by this physical quantity [39] and prefers the deconfinement phase [40,41]: $\langle\phi\rangle$ becomes finite according to the spontaneous breakdown of the $Z_{N_{c}}$ symmetry with the trivial holonomy. In contrast, if the symmetry is intact, one finds $\langle\phi\rangle=0$, indicating the confining phase with the non-trivial holonomy.

Thus, $\phi$ plays the role of an exact order parameter for the $\mathbb{Z}\left(N_{c}\right)$ symmetry for pure-glue QCD, in which the quark degree of freedom is decoupled according to its infinitely heavy mass. It is worth mentioning that the $\mathbb{Z}\left(N_{c}\right)$ symmetry is broken explicitly in the presence of dynamical quarks with finite mass, considering the anti-symmetric nature of fermions, resulting in that $\langle\phi\rangle$ is not an exact order parameter for $Z_{N_{c}}$ symmetry any more. However, from the phenomenological point of view, incorporating $\phi$ and dynamical quarks has been quite successful to a certain extent to explain various features of the QCD phases transition: The crossover near $T_{c}$ for instance for $N_{f}=2$. Hence, in the present talk as done in the pNJL model, we have incorporated the instanton-based model with the $\mathrm{SB} \chi \mathrm{S}$ and the traced Polyakov loop as an order parameter for the $\mathbb{Z}\left(N_{c}\right)$ symmetry. The trace over color space in Eq. (3) can be evaluated further in terms of $\phi$ and $\phi^{*}$ using Eq. (5) as follows:

$$
\begin{aligned}
\operatorname{Tr}_{c} \ln \left[\left(1+e^{-\frac{E_{\boldsymbol{k}, T}-i A_{4}}{T}}\right)\left(1+e^{-\frac{E_{\boldsymbol{k}, T}+i_{4}}{T}}\right)\right] & =\ln \left[1+N_{c}\left(\phi+\phi^{*} e^{-\frac{E_{\boldsymbol{k}, T}}{T}}\right) e^{-\frac{E_{\boldsymbol{k}, T}}{T}}+e^{-\frac{3 E_{\boldsymbol{k}, T}}{T}}\right] \\
& +\ln \left[1+N_{c}\left(\phi^{*}+\phi e^{-\frac{E_{\boldsymbol{k}, T}}{T}}\right) e^{-\frac{E_{\boldsymbol{k}, T}}{T}}+e^{-\frac{3 E_{\boldsymbol{k}, T}}{T}}\right] .
\end{aligned}
$$

On top of $\Omega_{\text {eff }}^{\mathrm{q}+\mathrm{A}_{4}}$ (now becoming $\Omega_{\mathrm{eff}}^{\mathrm{q}+\phi}$ ), an additional pure-glue effective thermodynamic potential was suggested in Refs. [8-11,14] as a function of $\phi$ and $\phi^{*}$ :

$$
\Omega_{\mathrm{eff}}^{\phi}=-T^{4}\left[\frac{b_{2}(T)}{2}\left(\phi \phi^{*}\right)+\frac{b_{3}}{6}\left(\phi^{3}+\phi^{* 3}\right)-\frac{b_{4}}{4}\left(\phi \phi^{*}\right)^{2}\right],
$$


where the coefficient $b_{2}$ is a function of $T$ :

$$
b_{2}(T)=a_{0}+a_{1}\left[\frac{T_{0}}{T}\right]+a_{2}\left[\frac{T_{0}}{T}\right]^{2}+a_{3}\left[\frac{T_{0}}{T}\right]^{3} .
$$

Here, the $T_{0}$ denotes critical $T$ in pure-glue QCD, resulting in $T_{0}=270 \mathrm{MeV}$ at which the first-order phase transition occurs. Note that $T_{0}$ is different from $T_{c}$, which will be computed later in the presence of the mixing of the dynamical quarks and $\phi$. The coefficients, $a$ and $b$, are listed in Table 1 [42]. This parameterization of the effective potential in Eq. (7) bears the $Z_{N_{c}}$ symmetry, conserved in pure-glue QCD by construction, and works qualitatively well up to $T \approx(2-3) T_{c}$, from which the transverse gluons come into play considerably.

\begin{tabular}{c|c|c|c|c|c}
$a_{0}$ & $a_{1}$ & $a_{2}$ & $a_{4}$ & $b_{3}$ & $b_{4}$ \\
\hline 6.75 & -1.95 & 2.63 & -7.44 & 1.0 & 7.50
\end{tabular}

Table 1. Coefficients for $\Omega_{\mathrm{eff}}^{\phi}$ in Eqs. (7) and (8), taken from Refs. [8-10].

Finally, substituting Eq. (6) into Eq. (3) and adding Eq. (7) to it, we arrive at the following expression for the effective thermodynamic potential with the two order parameters, $\sigma^{2}$ for the chiral phase and $\phi\left(\phi^{*}\right)$ for the deconfinement phase transitions, at finite $T$ and $\mu_{R}=0$ :

$$
\begin{aligned}
\Omega_{\mathrm{eff}} & =\Omega_{\mathrm{eff}}^{\mathrm{q}+\Phi}+\Omega_{\mathrm{eff}}^{\Phi}=2 \sigma^{2}-2 N_{f}\left[N_{c} \int \frac{d^{3} \boldsymbol{k}}{(2 \pi)^{3}} E_{\boldsymbol{k}, T}\right. \\
& +T \int \frac{d^{3} \boldsymbol{k}}{(2 \pi)^{3}} \ln \left[1+N_{c}\left(\Phi+\bar{\Phi} e^{-\frac{E_{\boldsymbol{k}, T}}{T}}\right) e^{-\frac{E_{\boldsymbol{k}, T}}{T}}+e^{-\frac{3 E_{\boldsymbol{k}, T}}{T}}\right] \\
& \left.+T \int \frac{d^{3} \boldsymbol{k}}{(2 \pi)^{3}} \ln \left[1+N_{c}\left(\bar{\Phi}+\Phi e^{-\frac{E_{\boldsymbol{k}, T}}{T}}\right) e^{-\frac{E_{\boldsymbol{k}, T}}{T}}+e^{-\frac{3 E_{\boldsymbol{k}, T}}{T}}\right]\right] \\
& -T^{4}\left[\frac{b_{2}(T)}{2}(\Phi \bar{\Phi})+\frac{b_{3}}{6}\left(\Phi^{3}+\bar{\Phi}^{3}\right)-\frac{b_{4}}{4}(\Phi \bar{\Phi})^{2}\right]
\end{aligned}
$$

where we have replaced the $\phi$ into its mean value $\langle\phi\rangle \equiv \Phi$ as done in Refs. [8-10]. Although, this expression for $\Omega_{\mathrm{eff}}$ is very similar to those given in Refs. [8-11,14], ours is distinctive from them quantitatively in several points:

i) The scale parameter of the model $\Lambda \approx 1 / \bar{\rho}$ is obtained as a function of $T$ by solving the instanton distribution function as discussed in the previous Section. Moreover, the $M$ is expressed as a function of $\boldsymbol{k}$ and $T\left(M_{\boldsymbol{k}, T}\right)$, rather than a constant, manifesting the partial chiral restoration and nontrivial QCD vacuum contributions.

ii) Consequently, there appears no divergence in the energy integral $\propto \int d^{3} \boldsymbol{k} E_{\boldsymbol{k}, T}$ in Eq. (9) by virtue of the $\boldsymbol{k}$-dependent $M$, which plays the role of an intrinsic ultraviolet (UV) regulator, being different from other local-interaction models, such as the usual pNJL model.

iii) At the smae time, as for the $2 N_{f}$-'t Hooft interaction, the quark-meson coupling strength depends on $k$ as well as $T$, being different from that in other models, in which it is a constant value fixed at zero $T$.

iv) All the relevant quantities at zero $T$, the $M_{0,0}, \sigma_{0}$ and $n_{0}$ for instance, are determined selfconsistently by solving the saddle-point equations.

Now, we evaluate the equations of motion with respect to the mean fields, $\sigma, \Phi$, and $\bar{\Phi}$, by minimizing $\Omega_{\text {eff }}$ :

$$
\frac{\delta \Omega_{\mathrm{eff}}}{\delta \Phi}=0, \quad \frac{\delta \Omega_{\mathrm{eff}}}{\delta \bar{\Phi}}=0, \quad \frac{\delta \Omega_{\mathrm{eff}}}{\delta \sigma}=0
$$



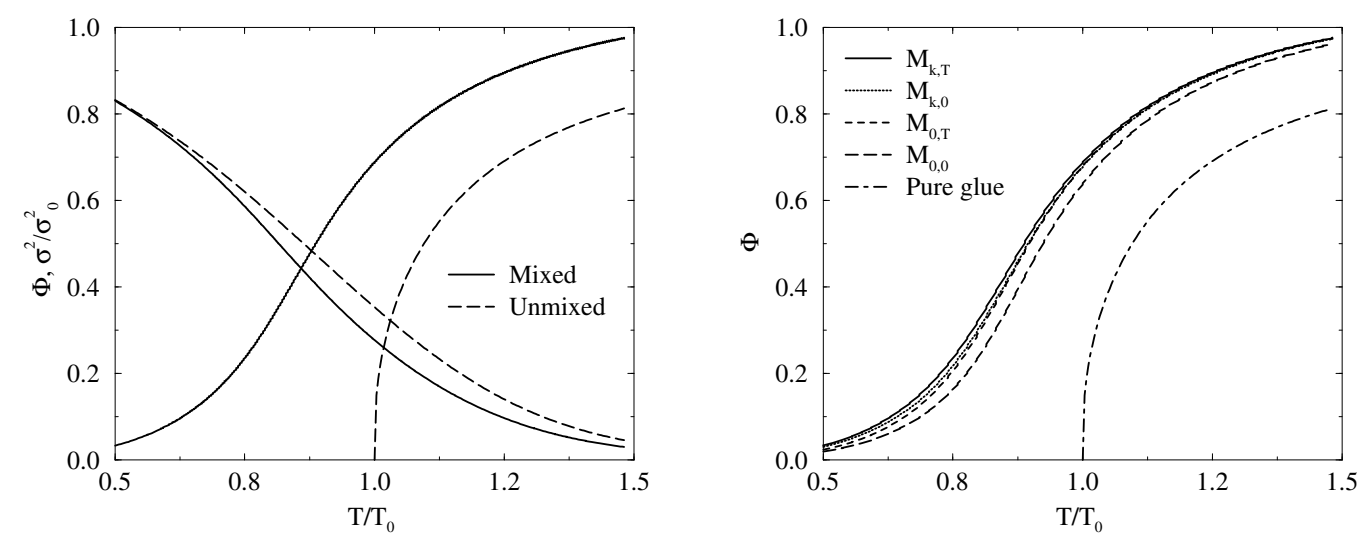

Fig. 1. Left: $\Phi$ (increasing curves) and normalizaed $\sigma^{2}$ (decreasing ones) for $T_{0}=270 \mathrm{MeV}$ using $M_{\boldsymbol{k}, T}$. The solid and dashed lines indicate the cases with and without the dynamical quark and $\Phi$ mixing, respectively. Right: $\Phi$ as a function of $T$ for $T_{0}=270 \mathrm{MeV}$, employing $M_{k, T}$ (solid), $M_{k, 0}$ (dotted), $M_{0, T}$ (dashed), and $M_{0,0}$ (long-dashed). We also draw $\Phi$ for the pure-glue case (dot-dashed).

From the first two equations, it can be easily seen that $\Phi=\bar{\Phi}$ at the saddle point, we can compute the values for the $\sigma^{2}$ and $\Phi$, resulting in the following asymptotic behaviors:

$$
\lim _{T \rightarrow 0}\left[2 \sigma^{2}\right]=N_{c} N_{f} \int \frac{d^{3} \boldsymbol{k}}{(2 \pi)^{3}} \frac{M_{\boldsymbol{k}, T}^{2}}{E_{\boldsymbol{k}, T}} \text { as } \Phi \rightarrow 0, \quad \lim _{T \rightarrow \infty}\left[2 \sigma^{2}\right]=0 \text { as } \Phi \rightarrow 1,
$$

showing appropriate asymptotic chiral behaviors as expected.

\section{Numerical results}

In this Section, we present the numerical results for the two different order parameters, $\sigma^{2}$ and $\Phi$ as functions of $T$, and related discussions are given as well. Hereafter, we will used the normalized value for $\sigma^{2}, \sigma^{2} / \sigma_{0}^{2}$ for convenience. Firstly, in the left panel of Figure 1, we draw them for the cases with (solid lines) and without (dashed lines) the mixing of the dynamical quark and $\Phi$, using $M_{k, T}$. Here, we employ $T_{0}=270 \mathrm{MeV}$ for the pure-glue potential in Eq. (7). In order for the unmixed case (dashed lines), in which the quarks are decoupled from the pure gluodynamics, we set $M$ infinite, equivalently $E_{\boldsymbol{k}, T} \rightarrow \infty$. As shown in the figure, even with or without the mixing, $\sigma^{2}$ showed the crossover for the chiral phase transition. On the contrary, the phase transition pattern for $\Phi$ turns out to be distinctive, depending on the mixing. In this sense, the crossover of $\Phi$ is caused by the mixing, as suggested by the pNJL models $[7-10,14]$ and shown by the LQCD simulations with dynamical quarks [6]. An interesting behavior shown in the left panel of Figure 1 is that the quark-gluon mixing effect increases then decreases for $\sigma^{2}$ as indicated in the solid and dashed lines. This tendency comes from the combination of the opposite behaviors of $\Phi$ (increasing) and $e^{-E_{k, T} / T}$ (decreasing) in $\sigma^{2}$.

In the right panel of Figure 1, we show the numerical results for $\Phi$ as a function of $T$ for different types of $M$, as listed in Table 2 . We again employed $T_{0}=270 \mathrm{MeV}$. $\Phi$ for the pure-glue potential, showing the first-order deconfinement phase transition, is also given in the right panel of Figure 1 for comparison. From the figure, it is clearly shown that the $T$ - and/or $\boldsymbol{k}$-dependent $M$ make $\Phi$ shifted to lower $T$, resulting in lowering $T_{c}$ (we will discuss the numerical values for $T_{c}$ later in detail).

For simplicity, we approximate it considering only the leading contributions as follows:

$$
T^{3}\left[b_{4} \Phi^{3}-b_{3} \Phi^{2}-b_{2}(T) \Phi\right] \approx 4 N_{c} N_{f} \int \frac{d^{3} \boldsymbol{k}}{(2 \pi)^{3}} e^{-E_{\boldsymbol{k}, T} / T} .
$$


EPJ Web of Conferences

\begin{tabular}{c|c|c|c}
$M_{\boldsymbol{k}, T}[\mathrm{MeV}]$ & $M_{\boldsymbol{k}, 0}[\mathrm{MeV}]$ & $M_{0, T}[\mathrm{MeV}]$ & $M_{0,0}[\mathrm{MeV}]$ \\
\hline$M_{0, T}\left[\frac{2}{2+\boldsymbol{k}^{2} \bar{\rho}^{2}}\right]^{2}$ & $M_{0,0}\left[\frac{2}{2+\boldsymbol{k}^{2} \bar{\rho}_{0}^{2}}\right]^{2}$ & $M_{0,0}\left[\frac{\sqrt{n} \bar{\rho}^{2}}{\sqrt{n_{0}} \bar{\rho}_{0}^{2}}\right]$ & 350
\end{tabular}

Table 2. Notations for $M$ given in Figure 1.

When we take into account the $T$ - and/or $\boldsymbol{k}$-dependent $M$, the strength of $M$ decreases as $\boldsymbol{k}$ and/or $T$ increases, and the same for $E_{\boldsymbol{k}, T}$. As a result, the integrand in the r.h.s. of Eq. (12) tends to be larger, as $k$ and/or $T$ increases, than that with a constant $M, M_{0,0} \approx 350 \mathrm{MeV}$. To make things clear, we put $T=T_{0}$ in Eq. (12) for example, then have

$$
T_{0}^{3}\left[b_{4} \Phi^{3}-b_{3} \Phi^{2}\right] \approx \underbrace{4 N_{c} N_{f} \int \frac{d^{3} \boldsymbol{k}}{(2 \pi)^{3}} e^{-E_{\boldsymbol{k}, T_{0}} / T_{0}}}_{A}>\underbrace{4 N_{c} N_{f} \int \frac{d^{3} \boldsymbol{k}}{(2 \pi)^{3}} e^{-E_{0,0} / T_{0}}}_{B},
$$

where we have used the notation, $E_{0,0}^{2}=M_{0,0}^{2}+\boldsymbol{k}^{2}$. In order to satisfy the relations of Eq. (13), $\Phi$ for $A, \Phi_{A}$ must be bigger than $\Phi_{B}$ at $T \stackrel{,=}{=} T_{0}$, since $\Phi$ is positive definite and $b_{4} \gg b_{3}$. This observation is also true for arbitrary $T$, except for the limiting cases, $T \rightarrow 0$ or $\infty$. Ats the same time, therefore, this situation can be understood as that $\Phi$ is shifted downward almost horizontally: $T_{c}$ is lowered consequently.

However, this lowering $T_{c}$ behavior must be understood separately for the $k$ and $T$ dependences in $M$, since the both curves with $M_{0, T}$ and $M_{k, 0}$ show it, as depicted in the right panel of Figure 1 .

i) $T$ dependence: $M_{0, T}$

It tells us that the partial chiral restoration, indicated by decreasing $M \propto \bar{\rho}^{2}$ with respect to $T$, has effects on the deconfinement phase transition, although these two phase-transition mechanisms are believed to be different to each other. Here is a microscopic explanation for this: As $T$ increases, the pseudo-particle (instanton) become smaller in its size $\sim \bar{\rho}$, decreasing QCD vacuum contribution simultaneously, resulting in that the quarks are enable to travel more freely with less interactions with the instantons, and strings attached to each quark are extended more at lower string tension, in comparison to the case without the partial chiral restoration in $M$. Hence, the condensation of the strings can happen easily at lower $T$, toward the deconfinement phase.

ii) $\boldsymbol{k}$ dependence: $M_{\boldsymbol{k}, 0}$

The $k$ dependence in $M$ is originated from the delocalization of quark fields in the presence of the instanton background [33], not from $T$-related effects. As momentum transfer increases, quarks become lighter $\propto 1 / \boldsymbol{k}^{4}$, loosing the nontrivial QCD vacuum contributions. In other words, the instanton effect is weakened seemingly in the $k$ integrals. Consequently, being similar to the $T$-dependence case, the deconfinement phase transition takes place at lower $T$. From these explanations, one is led to a conclusion that the QCD vacuum contributions and the partial chiral restoration play considerable roles for the deconfinement phase transition to a certain extent in the presence of the mixing.

Now, we are in a position to calculate the critical temperatures $T_{c}$ for the crossover transitions numerically. They are determined by the chiral and Polyakov susceptibilities as in Ref. [7]. Being almost equivalently, they can be also obtained from the maximum values of $\partial \Phi / \partial T$ and $\partial \sigma^{2} / \partial T$ for the deconfinement and chiral phases, respectively as in Refs. [8-10,45]. In the present talk, we employ the later method. In Table 3, we list them for $\Phi$ and $\sigma^{2}$, assigned as $T_{c}^{\mathbb{Z}}$ and $T_{c}^{\chi}$, respectively, for each type of $M$. Note that we do not show the numerical results for $T_{c}^{\chi}$ for the cases with $M_{0, T}$ and $M_{0,0}$, since they are UV divergent, proportional to $\int \boldsymbol{k}^{3} d \boldsymbol{k}$, unless a cutoff is introduced by hand.

As discussed previously, from the table, one can see clearly that $T_{c}$ is lowered by inclusion of the $(k, T)$ dependence in $M$. It turns out that the shift of $T_{c}^{\mathbb{Z}}$ is about $10 \%,(240 \rightarrow 227) \mathrm{MeV}$ for $M_{0,0} \rightarrow M_{k, T}$. Interestingly, if we take into account full $(k, T)$ dependence, $T_{c}^{\mathbb{Z}}$ and $T_{c}^{\chi}$ get closer to each other as shown in Table $3,\left(T^{\Phi}, T^{\sigma^{2}}\right)=(227,216) \mathrm{MeV}$. About $5 \%$ discrepancy $(\sim 10 \mathrm{MeV})$ is observed between them, showing a tendency $T_{c}^{\chi}<T_{c}^{\mathbb{Z}}$. By turning off the $T$ dependence in $M\left(M_{k, 0}\right)$, the discrepancy between $T_{c}^{\chi}$ and $T_{c}^{\mathbb{Z}}$ becomes larger up to about $15 \%,\left(T^{\Phi}, T^{\sigma^{2}}\right)=(225,265) \mathrm{MeV}$. For interpreting this behavior, we take a look on the $T$ dependence of $\sigma^{2}$ for the cases with $M_{\boldsymbol{k}, T}$ and $M_{\boldsymbol{k}, 0}$, 


\begin{tabular}{c|c|c|c}
$M_{k, T}[\mathrm{MeV}]$ & $M_{\boldsymbol{k}, 0}[\mathrm{MeV}]$ & $M_{0, T}[\mathrm{MeV}]$ & $M_{0,0}[\mathrm{MeV}]$ \\
\hline \hline$T_{c}^{\mathrm{Z}}=227$ & $T_{c}^{\mathrm{Z}}=225$ & $T_{c}^{\phi}=230$ & $T_{c}^{\phi}=240$ \\
\hline$T_{c}^{\sigma^{2}}=216$ & $T_{c}^{\sigma^{2}}=265$ & $\cdots$ & $\cdots$
\end{tabular}

Table 3. $T_{c}$ computed from $\Phi$ and $\sigma^{2} / \sigma_{0}^{2}$ for $T_{0}=270 \mathrm{MeV}$.
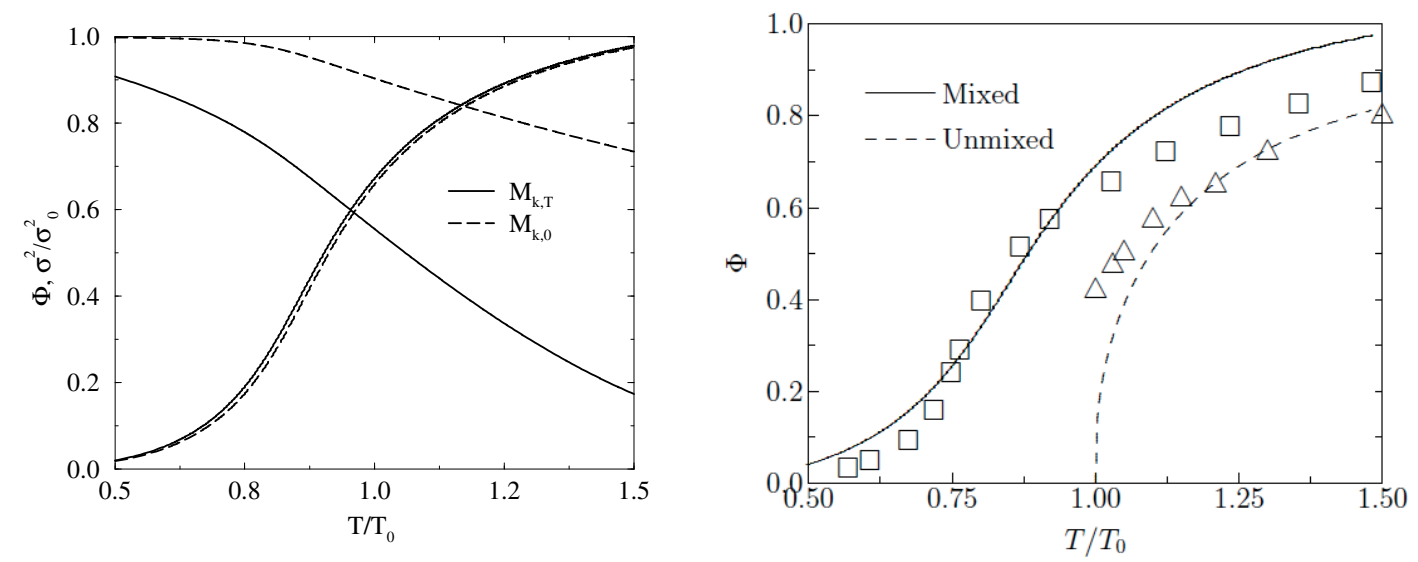

Fig. 2. Left: $\Phi$ (increasing curves) and $\sigma^{2} / \sigma_{0}^{2}$ (decreasing ones) as functions of $T$ for $T_{0}=270 \mathrm{MeV}$, employing $M_{k, T}$ (solid) and $M_{k, 0}$ (long-dashed). Right: $\Phi$ as a function of $T$ for $T_{0}=270$. The solid and dashed lines indicate the cases with and without the dynamical quark and $\Phi$ mixing, respectively. The notations $\square$ and $\triangle$ indicate the full and quenched lattice data, respectively, taken from Refs. [46] and [47].

and draw the numerical results in the left panel of Figure 2. For comparison, we also draw $\Phi$ for the two cases. Note that $\sigma^{2}$ show obvious difference for the cases with $M_{k, T}$ and $M_{k, 0}$. This is the reason why the discrepancy between $T_{c}^{\mathbb{Z}}$ and $T_{c}^{\chi}$ is so large for $M_{k, 0}$. At the same time, this strong dependence on $T$ for the chiral phase transition interprets the larger shift of $T_{c}^{\chi}$, $(265 \rightarrow 216) \mathrm{MeV}$. From this observation, we can conclude that the $T$ dependence in $M$ plays an important role in exploring chiral phase transition at finite $T$.

It is worth mentioning that, from the LQCD analyses, it turned out that $T_{c}^{\chi} \approx 180 \mathrm{MeV}$ for $N_{f}=2$ using the clover-improved Willson fermions [4]. Also, using the renormalization-group (RG) improved action, it was found that $T_{c}^{\chi} \approx 171 \mathrm{MeV}$ [5]. These values are significantly smaller than ours by $(10-20) \%$. If this is the case, one may need more strong $T$ dependence for $M$ in the present approach, since the $T$ dependence of $\sigma^{2}$ is mainly governed by the behavior of $M$. In other words, the instanton effects must decrease much faster as $T$ increases. There can be several possible scenarios to satisfy this condition:

i) If we consider a correct instanton distribution function, rather than the simplified one, according to the large- $N_{c}$ limit, $T_{c}^{\chi}$ may be lowered. To test this, we have the ratio of $M$ computed with the correct one:

$$
\frac{M_{\text {correct }}}{M_{0, T}}=\frac{2 \mathcal{F}^{5 / 2}}{3 \sqrt{\pi} \bar{\rho}^{2}} \int d \rho \rho^{6} e^{-\mathcal{F} \rho^{2}}=(0.46-0.47)
$$

We, however, verify that this modification does not work for lowering $T_{c}^{\chi}$, whereas the strength of $M$ is reduced approximately by half.

ii) Additional $T$-dependent terms can be taken into account. For instance, we assumed that the Lgrangian multiplier $\lambda$ is independent on $T$ in deriving $M_{k, T}$. If it has the $T$ dependence, we can modify $M_{k, T}$ as follows:

$$
M_{k, T} \rightarrow \sqrt{\frac{\lambda}{\lambda_{0}}} M_{k, T}
$$




\section{EPJ Web of Conferences}

Quantitatively, the $T$-dependent $\lambda$ can not be determined self-consistently within the framework. From a very rough estimation, based on the assumption that $n_{0}$, which is the instanton packing fraction at $T=0$, converges with a brute cutoff $\Lambda \approx 1 / \bar{\rho}_{0}$, we can obtain a relation $\lambda_{0} \propto 1 / \bar{\rho}_{0}^{2}$ [33]. Using this assumption, the expression for $M_{k, T}$ is modified into

$$
M_{0, T} \rightarrow M_{0,0} \sqrt{\frac{\lambda}{\lambda_{0}}}\left[\frac{\sqrt{n} \bar{\rho}^{2}}{\sqrt{n_{0}} \bar{\rho}_{0}^{2}}\right]=M_{0,0}\left[\frac{\sqrt{n} \bar{\rho}}{\sqrt{n_{0}} \bar{\rho}_{0}}\right] .
$$

It turns out that this rough assumption makes things worse: $T_{c}^{\chi}$ is shifted to a larger value as expected in Eq. (16).

iii) We may not ignore the Matsubara frequency $w_{n}$ in the denominator. In the presence of the mixing, $\Phi$ may provide effects on the instanton distribution function, becoming an exponentially decreasing function, not a gaussian one. We, however, do not perform quantitative calculations for these possibilities here and leave them for future works.

iv) Additional $T$ dependence can be added to the instanton distribution function, according to the fermion overlap matrix, if one considers full QCD in computing the distribution function [43], being different from the present talk based on the variational method in pure-glue QCD (the HarringtonShepard caloron) [31]. Moreover, the instanton clustering, which was suggested as a main contribution for the chiral phase transition $[43,44]$ and not taken into account here, may be responsible for lowering $T_{c}^{\chi}$.

v) We note that $T_{0}$ for the pure-glue potential can be chosen as a smaller value than $270 \mathrm{MeV}$, which has been used throughout in the present talk. As shown in Refs. [8-10], by taking $T_{0}=190 \mathrm{MeV}$, the computed values for $T_{c}^{\chi}$ became compatible with those from the LQCD simulations. Although we have not presented detailed results for lower $T_{0}$, we could obtain $T_{c}^{\chi}=194 \mathrm{MeV}$ using $M_{k, T}$ at $T_{0}=200$ $\mathrm{MeV}$, showing about $10 \%$ decreasing.

As for $T_{c}^{\mathbb{Z}}$ estimated in the LQCD, using the clover-improved Wilson fermions similarly, it was determined about $210 \mathrm{MeV}$ [6], which is rather compatible with ours. It was observed in the usual local pNJL model [8-10] that $T_{c}$ taken from the two different order parameters are almost consistent: $T_{c}^{\chi} \approx T_{c}^{\mathbb{Z}} \approx 220 \mathrm{MeV}$ for $T_{0}=270 \mathrm{MeV}$. It turned out that $T_{c}^{\mathbb{Z}}=215 \mathrm{MeV}$ in Ref. [45] with pNJL.

Finally, we compare our results for $\Phi$ with the LQCD data from Refs. [46] and [47] in the right penal of Figure 2, in which a full and quenched calculations were done for $N_{f}=2$. In their works, it was observed that $T_{c}^{\mathbb{Z}}=202 \mathrm{MeV}$, which is about $10 \%$ lower than ours, $227 \mathrm{MeV}$ for $N_{f}=2$, and 270 $\mathrm{MeV}$ for $N_{f}=0$. As shown in the figure, the LQCD data, indicated by $\square$ (full) and $\triangle$ (quenched), are in a qualitative agreement with the present results, but not quantitative. Especially, our result for the mixed case deviates much from it for the region $T>T_{0}$.

\section{Summary and outlook}

In the present talk, we have attempted to derive an effective thermodynamic potential $\Omega_{\mathrm{eff}}$ at finite $T$ and zero quark-chemical potential $\left(\mu_{\mathrm{R}}=0\right)$ in the chiral limit. We restricted ourselves to $N_{c}=3$ and $N_{f}=2$. Motivated by the Polyakov-loop-augmented Nambu-Jona-Lasinio model, we wanted to incorporate two different order parameters, $\sigma^{2}$ and $\Phi$, which characterize the chiral and deconfinement phase transitions, respectively.

In order to discuss the spontaneous breakdown of chiral symmetry at finite $T$, we employed the singular-gauge instanton solution, and the fermionic Matsubara formula to express the effective chiral action as a function of $T$. We employed the instanton-distribution function, derived from the Harrington-Shepard caloron, to obtain the instanton density and average instanton size as functions of $T$. It turned out that these two quantities decreased but finite, indicating that the instanton effect survives even beyond $T_{c}$ [31]. The $(\boldsymbol{k}, T)$-dependent $M, M_{\boldsymbol{k}, T}$ was derived in the large- $N_{c}$ limit. We found that the $\boldsymbol{k}$ dependence of $M$ becomes weaker as $T$ increases. At the same time, the absolute value of $M_{k, T}$ was also reduced with respect to $T$. To include the Polyakov loop as an order parameter for the $\mathbb{Z}\left(N_{c}\right)$ symmetry, we took into account imaginary quark-chemical potential $\mu_{I} \equiv A_{4}$, which was identified as the traced Polyakov loop $\Phi$. Combining all these ingredients, we could construct $\Omega_{\text {eff }}$ with an 
additional pure-glue $\mathrm{SU}\left(N_{c}\right)$ gauge potential. By minimizing $\Omega_{\mathrm{eff}}$ with respect to external fields such as $\sigma$ and $\Phi$, we could compute $\sigma^{2}$ and $\Phi$ as functions of $T$ numerically. From the various numerical results we have found the followings:

i) In the presence of the mixing of the dynamical quarks and $\Phi$, we observed that $\Phi$ is very sensitive to the mixing, showing the crosssover and first-order transitions with and without it, respectively. In contrast, $\sigma^{2}$ is insensitive to it, indicating the crossover phase transition.

ii) $M$ was expressed as a decreasing function of $T$ as well as $k$. Due to this, $T_{c}^{\mathbb{Z}}$ was lowered by about (5-10)\%, in comparison to that with constant mass $M_{0,0} \approx 350 \mathrm{MeV}$. From this observation, we explain this lowering $T_{c}^{\mathbb{Z}}$ by that the nontrivial QCD vacuum contributions and partial chiral restoration play a significant role even in the deconfinement phase transition.

iii) If the $(k, T)$ dependence had been fully taken into account, we found that $T_{c}^{\mathbb{Z}}=227 \mathrm{MeV}$ and $T_{c}^{\chi}=216 \mathrm{MeV}$. The discrepancy between them became about $10 \mathrm{MeV}$, which was rather larger than that computed in the pNJL model. We also note that the LQCD simulations presented smaller $T_{c}^{\chi}$ than ours, whereas $T_{c}^{\mathbb{Z}}$ was compatible.

iv) Finally, we observed that $\sigma^{2}$ was depending much on $T$. Again, the partial chiral restoration turned out to be crucial to make proper results for the chiral phase transition.

Consequently, from the present talk, we could learn that the partial chiral restoration and nontrivial QCD vacuum effects must be taken into account appropriately to investigate the breaking patterns of the chiral and $\mathbb{Z}\left(N_{c}\right)$ symmetries at finite $T$. As a next step, we attempt to include the finite quarkchemical potential $\left(\mu_{R}\right)$, giving a full description for the QCD phase diagram on the $\mu_{R}-T$ plane. In addition to it, the finite current-quark mass, $m$ is also under consideration beyond the chiral limit. This is important, since the explicit breakdown of the flavor SU(3) symmetry modifies the QCD phase diagram to a good extent. However, including finite $m$ into the present framework has a huddle: One needs to consider the meson-loop corrections, which make significant modification on the physical quantities such as the chiral susceptibility, in comparison to those in the chiral limit [36]. Related works has been published [48], and are under progress and appear elsewhere.

\section{Acknowledgment}

This report was prepared as a proceeding for the international workshop Hadron Nuclear Physics (HNP) 2011, 21 - 24 February 2011, Pohang, Republic of Korea. The author is grateful to C. W. Kao, H. -Ch. Kim, and B. G. Yu for fruitful discussions. This work was supported by the grant NRF-20100013279 from National Research Foundation (NRF) of Korea. This work was also partially supported by the Grant of NSC96-2112-M033-003-MY3 from the National Science Council (NSC) of Taiwan.

\section{References}

1. M. G. Alford, A. Kapustin and F. Wilczek, Phys. Rev. D 59 (1999) 054502.

2. S. Hands, J. B. Kogut, M. P. Lombardo and S. E. Morrison, Nucl. Phys. B 558 (1999) 327.

3. Z. Fodor and S. D. Katz, Phys. Lett. B 534 (2002) 87.

4. Y. Maezawa et al., J. Phys. G 34 (2007) S651.

5. A. Ali Khan et al. [CP-PACS Collaboration], Phys. Rev. D 63 (2001) 034502.

6. V. G. Bornyakov et al. [DIK Collaboration], Phys. Rev. D 71 (2005) 114504.

7. K. Fukushima, Phys. Lett. B 591 (2004) 277.

8. C. Ratti and W. Weise, Phys. Rev. D 70 (2004) 054013.

9. C. Ratti, M. A. Thaler and W. Weise, Phys. Rev. D 73 (2006) 014019.

10. C. Ratti, M. A. Thaler and W. Weise, arXiv:nucl-th/0604025.

11. H. Hansen et al., Phys. Rev. D 75 (2007) 065004.

12. H. Kiuchi and M. Oka, Prog. Theor. Phys. 114 (2005) 813.

13. B. Vanderheyden and A. D. Jackson, Phys. Rev. D 64 (2001) 074016.

14. Y. Sakai, K. Kashiwa, H. Kouno and M. Yahiro, Phys. Rev. D 77 (2008) 051901.

15. I. Sachs and A. Wipf, Helv. Phys. Acta 65 (1992) 652. 
16. H. Boschi-Filho and C. P. Natividade, Mod. Phys. Lett. A 7 (1992) 3303.

17. S. V. Molodtsov and G. M. Zinovjev, Phys. Part. Nucl. Lett. 4 (2007) 11.

18. T. Schafer and E. V. Shuryak, Phys. Rev. D 53 (1996) 6522.

19. T. M. Schwarz, S. P. Klevansky and G. Papp, Phys. Rev. C 60 (1999) 055205.

20. T. C. Kraan and P. van Baal, Nucl. Phys. B 533 (1998) 627.

21. K. M. Lee and C. h. Lu, Phys. Rev. D 58 (1998) 025011.

22. D. Diakonov and N. Gromov, Phys. Rev. D 72 (2005) 025003.

23. D. Diakonov, N. Gromov, V. Petrov and S. Slizovskiy, Phys. Rev. D 70 (2004) 036003.

24. D. Diakonov, Acta Phys. Polon. B 39 (2008) 3365.

25. S. Slizovskiy, Phys. Rev. D 76 (2007) 085019.

26. E. M. Ilgenfritz et al., Phys. Rev. D 66 (2002) 074503.

27. M. Garcia Perez et al., Phys. Rev. D 60 (1999) 031901.

28. F. Lenz, J. W. Negele and M. Thies, Phys. Rev. D 69 (2004) 074009.

29. J. W. Negele, F. Lenz and M. Thies, Nucl. Phys. Proc. Suppl. 140 (2005) 629.

30. B. J. Harrington and H. K. Shepard, Nucl. Phys. B 124 (1977) 409.

31. D. Diakonov and A. D. Mirlin, Phys. Lett. B 203 (1988) 299.

32. S. i. Nam, J. Phys. G 37075002 (2010).

33. D. Diakonov, Prog. Part. Nucl. Phys. 51 (2003) 173.

34. K. Goeke, M. M. Musakhanov and M. Siddikov, Phys. Rev. D 76 (2007) 076007.

35. S. i. Nam and H. -Ch. Kim, Phys. Lett. B 647 (2007) 145.

36. S. i. Nam, Phys. Rev. D 79 (2009) 014008.

37. D. Diakonov, M. V. Polyakov and C. Weiss, Nucl. Phys. B 461 (1996) 539.

38. T. Schafer and E. V. Shuryak, Rev. Mod. Phys. 70 (1998) 323.

39. D. J. Gross, R. D. Pisarski and L. G. Yaffe, Rev. Mod. Phys. 53 (1981) 43.

40. A. M. Polyakov, Nucl. Phys. B 120 (1977) 429.

41. A. M. Polyakov, Phys. Lett. B 72 (1978) 477.

42. G. Boyd et al., Nucl. Phys. B 469 (1996) 419.

43. E. M. Ilgenfritz and E. V. Shuryak, Nucl. Phys. B 319 (1989) 511.

44. E. M. Ilgenfritz and E. V. Shuryak, Phys. Lett. B 325 (1994) 263.

45. S. Roessner, T. Hell, C. Ratti and W. Weise, Nucl. Phys. A 814 (2008) 118.

46. O. Kaczmarek and F. Zantow, Phys. Rev. D 71 (2005) 114510 .

47. O. Kaczmarek, F. Karsch, P. Petreczky and F. Zantow, Phys. Lett. B 543 (2002) 41.

48. S. i. Nam and C. W. Kao, Phys. Rev. D 82 (2010) 096001. 